\title{
Evaluation of Achilles Tendon Injuries by Ultrasonography and Magnetic Resonance Imaging (MRI)
}

M.M.Refat, H.M.T.Khater and N.H.Hamad

Radiogiagnosis Dept., Faculty of Medicine, Benha Univ., Benha, Egypt

E-Mail:mohamedsdgh73@gmail.com

\begin{abstract}
Achilles tendon disorders are a common health problem in middle-aged active people. With increasing sport activities in the general population, the number of overuse injuries has increased. Tendon disorders comprise $30-50 \%$ of all sports-related injuries. To evaluate the Injuries of Achilles tendon by ultrasonography and magnetic resonance imaging MRI. A cross sectional study was conducted including 20 patients complaining from distal leg pain with history of trauma to lower leg in Achilles tendon to be evaluated by ultrasonography and further evaluation by magnetic resonance imaging. The duration of the study was from 6 to 12 months. The mean age of cases was $41.80 \pm 10.7$ with range of (20-50) years, 50\% was in age group of (41-50) and percent of females was $45 \%$ and males was $55 \%$. Ultrasonography is an initial imaging of choice for evaluation of symptomatic Achilles tendon disorders. Diagnostic sensitivity reaches $91.1 \%$. However, Tendons that appeared normal by US should be followed by MRI for more diagnostic accuracy, detailed regional evaluation and subsequently can exclude other etiologies giving similar clinical manifestations.
\end{abstract}

Keywords: Achilles Tendon Injuries, Ultrasonography, Magnetic Resonance Imaging .

\section{Introduction}

The Achilles tendon is the most frequently injured ankle tendon. It lacks a tendon sheath; however it has a peritenon whose vascular system extends both within and outside the tendon [1].

Achilles tendon injuries may be classified as non insertional or insertional. The former group includes acute and chronic peritendinosis, tendinosis and rupture $2-6 \mathrm{~cm}$. a bove the insertion of the tendon on the calcaneus. The latter group includes Tendinosis which may be associated with Haglund deformity of the calcaneus [1].

Multiple imaging modalities have been used to diagnose Achilles tendon injuries, which include plain radiography, magnetic resonance imaging (MRl), and ultrasound. Each test has its own advantages and disadvantages. MRI \& US have been widely used in confirming the diagnosis of Achilles tendon injuries [2].

Ultrasound is a rapid, widely available and inexpensive modality for evaluation of the ankle tendon.The most common limitation of ultrasound is unfamiliarity with the technique and pathologic condition at us [3].

MRI is an excellent technique for those cases where the diagnosis--is uncertain; it is the most suitable for assessment of bone and soft tissue for persistent pain following injury [4].

Achilles tendon rupture is the most common tendon rupture in the lower extremity. The injury most commonly occurs in adults in their third to fifth decade of life. Acute ruptures often present with sudden onset of pain associated with a (snapping) or audible pop heard at the site of injury patient can describe the sensation of being kicked in the lower leg. The injury is causes significant pain and disability in patient population. [5]
Achilles tendon injuries typically occur in individuals who are only active intermittently (i.e. the weekend warrior althetes). The injury is reportedly misdiagnosed as an ankle sprain in $20 \%$ to $25 \%$ of patients. Moreover, patients in their third to the fifth decade of life are most commonly affected as $10 \%$ report a history of prodromal symptoms and known risk factors include prior intratendinuos degeneration (i.e. tendinosis),fluoroquinolone use. Steroid injection and inflammatory arthritides. [6].

The aim of this work is to evaluate the Injuries of Achilles tendon by ultrasonography and magnetic resonance imaging MRI.

\section{Patients and methods}

This study was carried out in 2019, A cross sectional study. Informed consent was obtained from all participants(including cases and controls) after being informed about the aims and process of the study as well as applicable objectives. The study had been approved by the local ethics committee on research involving human subjects of Benha faculty of Medicine.

\subsection{Study setting}

All patients were selected from the Radiogiagnosis Department of Salah EL-Din General Hospital. The study was include 20 patients complaining from (distal leg pain) with history of trauma in lower leg in Achilles tendon to be evaluated by ultrasonography and further evaluation by MRI

\subsection{Methods of the study}

Patients that fulfill the inclusion criteria had been subjected by: History Taking,

Clinical Examination, Ultrasonographic Examination and MRI. 
Ultrasonographic Examination All patients had standardized ultrasonography of Ultrasound examinations were performed using one of the following devices: GE Logic pro6 (12 MHz). GE Logic 3 (12 MHz). The ultrasonographic examination began with the patient in the supine position. Longitudinal scanning of the ankle was first performed to get an overall view of the tibiotalar joint and to detect joint effusion or intra-articular loose bodies. Then, the ankle joint syndesmosis and anterior inferior tibio-fibular ligament (AITFL) were assessed on transverse plane at anterolateral aspect of the distal tibia. Finally, while the patient in the same position; individual evaluation of the extensor tendons of the ankle was performed in both longitudinal and transverse planes starting from medial to lateral tibialis anterior tendon(TA), then extensor hallucis longus tendon( EHL), and most laterally, extensor digitoum Longus tendon( EDL). There after, slight inversion of the foot was performed while the patient in the same position to examine the lateral collateral ligaments and peroneal tendons. The anterior talofibular ligament (ATFL) was first examined in oblique transverse plane from the tip of lateral malleolus, anteromedially and slightly downwards, till the talus. Then, the calcaneofibular ligament (CFL) was examined in oblique longitudinal plane form the lateral malleolar tip downwards and slightly backwards to the lateral surface of the calcaneus. Regarding the peroneal tendons, they were examined from their supramalleolar musculotendinous junction, then just behind the lateral malleolus till their inframalleolar course in both longitudinal and transverse planes. Dynamic examination was obtained in eversion and dorsiflexion position to detect tendon dislocation or subluxation. The patient was then asked to laterally rotate the lower limb while lying supine to examine the deltoid ligament (DL) and flexor tendons. The former was examined in longitudinal scanning from its origin in the tip of the medial malleolus till its insertion into the talus, calcaneus, and navicular bones. The ankle flexor tendons were examined similar to the extensor tendons in longitudinal and transverse planes from medial to posterolateral: tibialis posterior tendon (TP), flexor digitorum longus tendon (FDL), and Finally the patient were asked to lie prone and rest on his/her toes. The Achilles tendon (AT): was examined from its musculotendinous junction to its calcaneal insertion in both longitudinal and transverse planes. PowerDoppler imaging was used to detect tissue hyperemia in cases of tendinopathy, enthesopathy, synovitis, and inflammatory conditions.

MRI Examination: All patients had MRI of the affected ankle(s) on a high field-strength scanners. MRI was performed using one of the following devices: GE Signa HDxt (1.5 T). Philips Achieva (1.5 T). Knee coil was used in all cases. Technique positioning every patient lied supine with the ankle and foot in neutral position, plantar flexion of 20 to 30 degrees has been advocated for reducing the "magic angle" artifact. No movement was allowed during examination by supporting the ankle using pads. Protocol The patients were examined by different pulse sequences including $\mathrm{T} 1, \mathrm{~T} 2$, proton density, gradient echo and short $\mathrm{T} 1$ inversion recovery (STIR). The examinations were done in different planes. We started examination by obtaining coronal localizers scout in order to have properly aligned sagittal images. Sagittal T1Wis for the ankle region were obtained at first. Sagittal images allow recognition of the proper plane of the ankle joint which is essential to adjust the Achilles tendon, articular cartilage, subtalar joint, tarsal sinus and plantar fascia. The second pulse sequence is to be obtained is the axial images in fast spin echo T2Wis. T2Wis in axial plane are demonstrating the bright signal of soft tissue edema, fluid in synovial sheath and joint effusion. The extension of this effusion outside the joint capsule is considered a strong indirect evidence of rupture of anterior talofibular. T1Wis are taken in the coronal planes. It allows further evaluation of the articular cartilage. The deltoid (DL) and the calcaneofibular (CFL) ligaments can also be evaluated properly at the coronal plane. STIR pulse sequence was done to detect abnormal marrow signal and to differentiate marrow edema (which appears very bright at STIR) from other lesions which appear hypointense in T1Wis such as focal sclerosis. The axial planes can visualize the talofibular and tibiofibular ligaments as well as the flexor and extensor tendons. Our usual protocol of examination was: Other parameters applied include slice thickness ranged from 3 to 5 mm, matrix $256 / 192$ or $512 / 224$, number of excitation 2 to 3 and field of view ranged from 12 to $16 \mathrm{~cm}$, better kept $<14 \mathrm{~cm}$.

Results obtained from the ultrasonographic examination were compared MRI examination.

\subsection{Statistical analysis}

Data collected throughout history, basic clinical examination, and outcome measures coded, entered and analyzed using Microsoft Excel software. Data were then imported into Statistical Package for the Social Sciences (SPSS version 20.0) (Statistical Package for the Social Sciences) software for analysis. According to the type of data qualitative represent as number and percentage, quantitative continues group represent by mean $\pm \mathrm{SD}$, the following tests were used to test differences for significance;. difference and association of qualitative variable , 
with one cell $<5$ by fisher test. Positive $=$ increase in the independent variable leads to increase in the dependent variable. Negative $=$ increase in the independent variable leads to decrease in the dependent variable.

\section{Results}

The mean age of cases was $41.80 \pm 10.7$ with range of (20-50) years, $50 \%$ was in age group of
(41-50) and percent of females was $45 \%$ and males was $55 \% .25 \%$ of cases had Post ankle pain induced by exercise. Table (1). $25 \%$ had Continuous pain and discomfort with walking, $30 \%$ had Stifness and limitation of movements in daily activities, $10 \%$ had Soft tissue thickening around the AT, 5\% had Pain started on jumping or landing on a dorsiflexed foot and $5 \%$ had Swollen and ecchymotic ankle. Fig (1).

Table (1) Demographic characteristics of the studied population

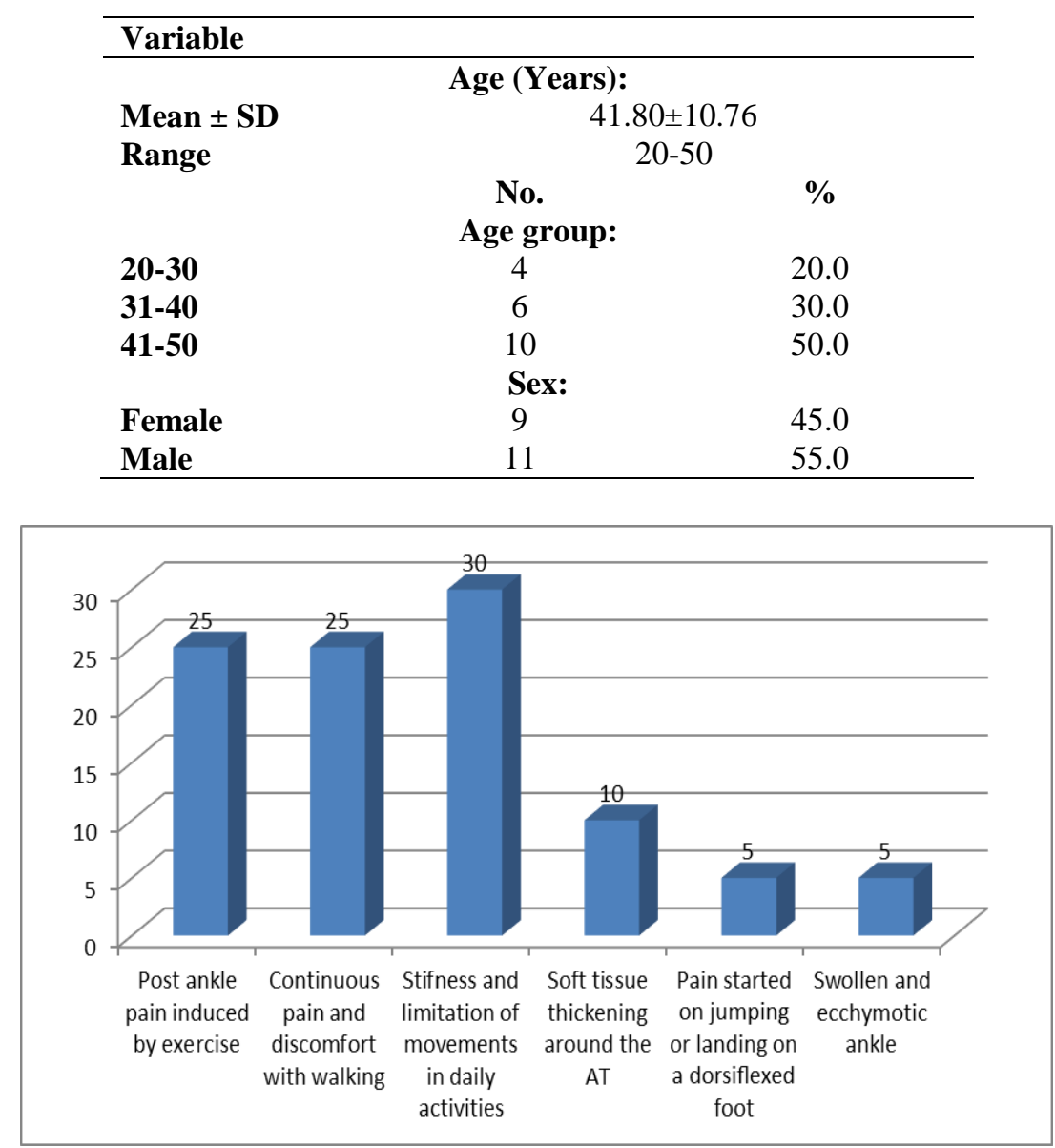

Fig (1) The clinical presentations of patients.

$20 \%$ of cases had tendinosis, $10 \%$ had Tenosynovitis, 55\% had Partial thickness tear, $15 \%$ had Full thickness tear. Table (2) and the side affected was The midportion of the tendon in $64.3 \%$ and The calcaneal insertion of the tendon in $35.7 \%$ of cases. Table (3).

Table (2) Classificaion of tendon pathlogy

\begin{tabular}{lll}
\hline Variable & No. & $\%$ \\
Pathlogy: & & \\
Tendinosis & 4 & 20.0 \\
Tenosynovitis & 2 & 10.0 \\
Partial thickness tear & 11 & 55.0 \\
Full thickness tear & 3 & 15.0 \\
\hline
\end{tabular}


Table (3) The side affected of the tendon in partial and complete tear cases

\begin{tabular}{lll}
\hline Variable & No. & $\%$ \\
\hline Side affected of the tendon: & 9 & 64.3 \\
The midportion of the tendon & 5 & 35.7 \\
The calcaneal insertion of the tendon & & \\
\hline
\end{tabular}

$33.3 \%$ of tendinopathy cases were swollen edematous tendon with heterogeneous echogenicity, $66.7 \%$ were Fluid surrounding the tendon sheath Table (4)

Table (4) The ultrasound features of tendinopathy

\begin{tabular}{lll}
\hline Variable & No. & $\%$ \\
\hline Ultrasound features: & & 33.3 \\
Swollen edematous tendon with heterogeneous echogenicity & 2 & 66.7 \\
Fluid surrounding the tendon sheath & 4 & \\
\hline
\end{tabular}

Amount of tendon retraction was $<1 \mathrm{~cm}$ in $10 \%$ of partial thickness tendon cases, A-P diameter of the tendon in the zone of abnormality was $9-10 \mathrm{~cm}$ in $90 \%$, hypoechoic zone was in $40 \%$ of casesTable (5).

Table (5) The ultrasound features of partial thickness tendon

\begin{tabular}{lll}
\hline Variable & $\begin{array}{l}\text { Partial } \\
\text { thickness tear }\end{array}$ & No. \\
\hline $\begin{array}{l}\text { The ultrasound features: } \\
\text { Amount of tendon retraction }\end{array}$ & & 10.0 \\
Posterior acoustic shadow & $-1 \mathrm{~cm}$ & \multicolumn{1}{c}{} \\
Kager fat herniation into the area of tendon abnormality & - & - \\
$\begin{array}{l}\text { A-P diameter of the tendon in the zone of abnormality } \\
\text { Localized disruption of tendon fibers }\end{array}$ & $9-10 \mathrm{~mm}$ & 9 \\
$\begin{array}{l}\text { Echogenicity of the tendon in the abnormal area (inaccurate) } \\
\text {-Hypoechoic }\end{array}$ & & \\
-Anechoic & Present & 40.0 \\
-Isoechoic & - & 4 \\
\hline
\end{tabular}

Amount of tendon retraction was $1 \mathrm{~cm}$ in $66.7 \%$ of full thickness tendon tear cases, A-P diameter of the tendon in the zone of abnormality was $9-10 \mathrm{~cm}$ in $90 \%$, hypoechoic zone was in $40 \%$ of cases. Table (6).

Table (6) The ultrasound features of full thickness tendon tear

\begin{tabular}{lll}
\hline Variable & Full thickness tear & No. \\
\hline The ultrasound features: & & $\%$ \\
Amount of tendon retraction & $1 \mathrm{~cm}$ & 66.7 \\
Posterior acoustic shadow & Present & 33.3 \\
Kager fat herniation into the area of tendon abnormality & Present & 33.3 \\
A-P diameter of the tendon in the zone of abnormality & - & \\
Localized disruption of tendon fibers & & \\
Echogenicity of the tendon in the abnormal area (inaccurate) & & 33.3 \\
-Hypoechoic & Present & 33.3 \\
-Anechoic & Present & 1 \\
-Isoechoic & & \\
\hline
\end{tabular}

On MRI examination, in 3 cases there were irregular areas of altered signal intensity in the preAchilles tendon fat pad (high T2 signal intensity), displaying low signal intensity in $\mathrm{T} 1$, high signal intensity in T2 and STIR, this was related to the edema of the tendon and we diagnosed the case as 
Achilles peritendinosis. Two cases show loss of the anterior concavity of the Achilles tendon in axial views and fusiform thickening of the tendon in sagittal views, in another case there were also small areas of increased $\mathrm{T} 1$ signal intensity within the tendon substance and altered signal intensity in $\mathrm{T} 2$ and STIR, these cases were diagnosed as Achilles tendinosis. Table (7).

Table (7) The MRI features of tendinopathy

\begin{tabular}{|c|c|c|c|}
\hline Variable & Diagnosis & & \\
\hline & & No. & $\%$ \\
\hline MRI features: & & & \\
\hline Areas of altered signal intensity in the pre-achilles fat & Peritendinosis & 3 & 100.0 \\
\hline pad & Tendinosis & 2 & 66.7 \\
\hline Loss of concavity & Tendinosis & 1 & 33.3 \\
\hline tendon(sagittal) & Tendinosis & 0 & 0.0 \\
\hline $\begin{array}{l}\text { increased T1 signal intensity of tendon substance } \\
\text { Altered signal intensity of Achilles tendon in T2 }\end{array}$ & & & \\
\hline
\end{tabular}

The MRI features of partial thickness tendon was Focal discontinuity of the fibers of the tendon in $45.4 \%$ of cases, Subcutaneous edema in
$36.6 \%$, Area of abnormal signal intensity in Kager fat in $18.2 \%$, Intratendinous hemorrhage in $9.1 \%$ fig (2).

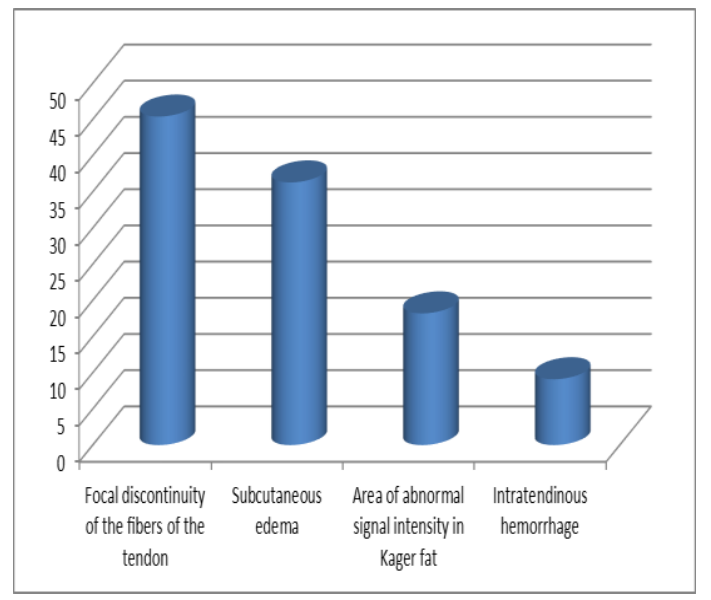

Fig (2) The MRI features of partial thickness tendon.

ROC curves showed Validity of ultrasound in compared to MRI in the diagnosis of Achilles tendon pathology, Fig (3).

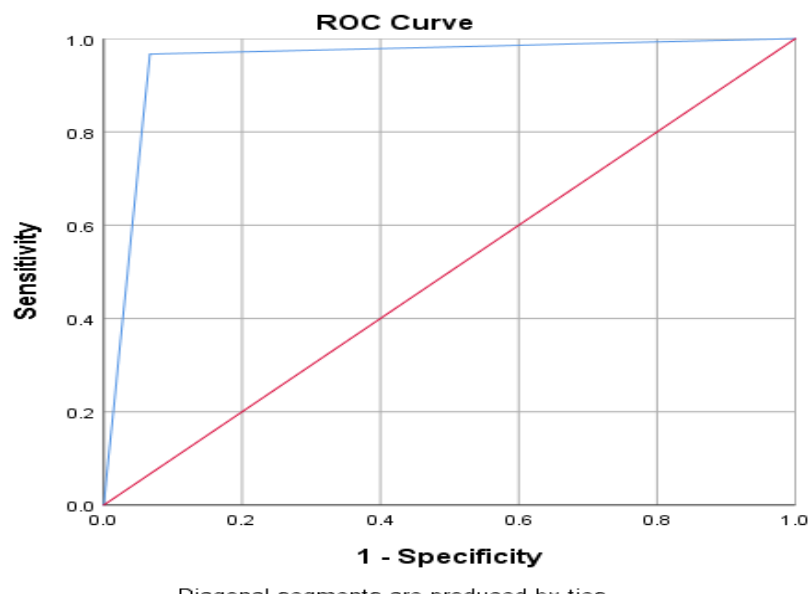

Diagonal segments are produced by ties.

Fig (3) ROC curves showed Validity of ultrasound in compared to MRI in the diagnosis of Achilles tendon pathology 


\section{Discussion}

The present study shows that $25 \%$ of cases had Post ankle pain induced by exercise, $25 \%$ had Continuous pain and discomfort with walking, $30 \%$ had Stifness and limitation of movements in daily activities, $10 \%$ had Soft tissue thickening around the AT, 5\% had Pain started on jumping or landing on a dorsiflexed foot and $5 \%$ had Swollen and ecchymotic ankle. 5\% of cases had Peritendinosis, $10 \%$ had Peritendinosis, $10 \%$ had Tenosynovitis, 55\% had Partial thickness tear, $15 \%$ had Full thickness tear.

Ibrahim et al [7] found that Achilles tendon was affected in 28 patients, 8 patients were diagnosed as tendinopathy, five of them were diagnosed as peritendinosis (as the Achilles tendon has no synovial sheath, it has a peritenon which is formed of connective tissue that enables smooth gliding of the tendon), tendinosis in three patients, partial thickness tear in 2 and full thickness tear in four patients.

The Achilles tendon is the strongest, largest and thickest tendon in the human body, measuring approximately $15 \mathrm{~cm}$ in length. The Achilles tendon originates in the mid-region of the leg and is formed by the junction of the two heads of the gastrocnemius muscle and the soleus muscle [8].

Although most Achilles tears occurs 2-6cm from its insertion, Achilles tears can be seen in two other locations: Distally and proximally. More common than an insertional tear, a proximal Achilles tear is, in reality, a musculotendinous junction injury [3].

Regarding the side affected of the tendon in partial and complete tear cases, the current study shows that the side affected was the midportion of the tendon in $64.3 \%$ and the calcaneal insertion of the tendon in $35.7 \%$ of cases.

Our results are in agreement with study of Ibrahim et al [7] as they reported that regarding the site affected in the tendon, in 12 cases the midportion of the tendon was affected (the region of avascularity) and in the remaining eight cases (from the total partial and complete tear), the region affected is at the calcaneal insertion.

To evaluate different treatment strategies in an objective way, imaging of the Achilles tendon can be helpful. Examinations of preference are MRI or ultrasonography. MRI of Achilles tendinopathy typically demonstrates a thickening of the tendon and a focal or diffusely raised intratendinous signal. Regions of increased signal on MR images and hypo-echoic areas on ultrasound have been shown to correspond to histopathological changes [9].

As regard the ultrasound features of tendinopathy, our study shows that $33.3 \%$ of tendinopathy cases were swollen edematous tendon with heterogeneous echogenicity, $66.7 \%$ were Fluid surrounding the tendon sheath.

Our results are in line with study of Ibrahim et al. [7] as they reported that After an ultrasound examination of the Achilles tendon, they found an increased amount of fluid around the Achilles tendon forming anechoic halo around it, the tendon has normal echogenicity, this was diagnosed as peritendinosis (five patients), three patients had fusiform swelling of the tendon which is hypoechoic with heterogeneous echogenicity and was diagnosed as tendinosis.

This coincided with Biancchi et al. [10] study in which they summarized the signs of tendinopathy in three main signs: increase the A$\mathrm{P}$ diameter of the tendon in the zone of the abnormality 9-10 $\mathrm{mm}$, swollen edematous tendon with heterogeneous echogenicity (tendinosis), and fluid surrounding the tendon (peritendinosis). However, the differentiation between tendinopathy and partial tear by US in their study is somehow inconclusive in some cases as they depended mainly on the increase in the A-P diameter of the tendon in the zone of abnormality which was present in 11 cases, the presence of localized disruption of tendon fibers which was diagnosed in 11 patients of partial tear, and hypoechoic area of abnormality in six patients. Three cases were missed as a result of moderate edema present and they could not accurately diagnose the disruption of tendon fibers, so further evaluation with MRI helped us to diagnose the cases.

Regarding the ultrasound features of partial thickness tendon, the present study shows that amount of tendon retraction was $<1 \mathrm{~cm}$ in $10 \%$ of partial thickness tendon cases, A-P diameter of the tendon in the zone of abnormality was 9$10 \mathrm{~cm}$ in $90 \%$, hypoechoic zone was in $40 \%$ of cases.

Our results are supported with study of Ibrahim et al., [7] as they reported in 16 patients of partial thickness tear, there was tendon retraction $<1 \mathrm{~cm}$ in one patient, increased A-P diameter of the tendon in the zone of abnormality $(9-10 \mathrm{~mm})$ in 11 patients, presence of localized disruption of some of the Achilles tendon fibers in 11 patients, hypoechoic area of abnormality in the tendon in six patients, however Kager fat herniation and posterior acoustic shadow are not seen in any of the cases of partial thickness tear. However, in three patients no disruption could be seen but was diagnosed by MRI as small partial tear.

As regard the ultrasound features of full thickness tendon, the current study shows that amount of tendon retraction was $1 \mathrm{~cm}$ in $66.7 \%$ of full thickness tendon tear cases, A-P diameter of the tendon in the zone of abnormality was 9- 
$10 \mathrm{~cm}$ in $90 \%$, hypoechoic zone was in $40 \%$ of cases.

Our results are supported with study of Ibrahim et al., [7] as they found that in the four patients of full thickness tear, they studied the amount of tendon retraction with the foot in neutral, dorsiflexion, and plantar positions, they found out the amount of tendon retraction increased in dorsiflexion position to be $1 \mathrm{~cm}$. This was present in all the four patients of full thickness tear, posterior acoustic shadow was present in two patients, Kager fat herniation in three patients, abnormal echogenicity of the area of the tendon in two patients, in one patient of these four cases there was a hypoechoic hematoma seen filling the tendon gap and was diagnosed as acute full thickness tear.

This also was in agreement with Hartgerink et al. [11]. Full thickness tear was diagnosed in four cases depending on the presence of a lot of sonographic signs as summarized in Hartgerink. The first amount of tendon retraction was $1 \mathrm{~cm}$ in two patients), presence of posterior acoustic shadowing, Kager fat herniation into the area of tendon abnormality, but the echogenicity of the tendon in the abnormal area was found inaccurate. They studied the amount of tendon retraction with the foot in neutral, dorsiflexion, and plantar flexion positions and they found out that it was increased with dorsiflexion position to be $1 \mathrm{~cm}$., this coincided with Fornge et al. 1998 , (in all the four patients) posterior acoustic shadow was seen in two patients, Kager fat herniation in three patients and abnormal area of echogenicity in the tendon substance in two patients, however in one of these four cases it was diagnosed as acute full thickness tear as a result of the presence of hypoechoic hematoma filling the tendon gap.

Regarding the MRI features of tendinopathy, our results show that on MRI examination, in 3 cases there were irregular areas of altered signal intensity in the pre-Achilles tendon fat pad (high T2 signal intensity), displaying low signal intensity in $\mathrm{T} 1$, high signal intensity in $\mathrm{T} 2$ and STIR, this was related to the edema of the tendon and we diagnosed the case as Achilles peritendinosis. Two cases show loss of the anterior concavity of the Achilles tendon in axial views and fusiform thickening of the tendon in sagittal views, in another case there were also small areas of increased $\mathrm{T} 1$ signal intensity within the tendon substance and altered signal intensity in T2 and STIR, these cases were diagnosed as Achilles tendinosis.

The MRI features of partial thickness tendon was Focal discontinuity of the fibers of the tendon in $45.4 \%$ of cases, Subcutaneous edema in $36.6 \%$, Area of abnormal signal intensity in
Kager fat in $18.2 \%$, Intratendinous hemorrhage in $9.1 \%$.

The MRI features of full thickness tendon was Full thickness disruption of the tendon fibers in $100 \%$, Hematoma seen within the tendon gap in $33.3 \%$.

In the study in our hands there is no significant difference between US and MRI as regard diagnosis of Achilles tendon pathology. Regarding Validity of ultrasound in compared to MRI in the diagnosis of Achilles tendon pathology, ROC curve shows that area under the curve 0.95 , sensitivity was $91.1 \%$, specificity was $100 \%$ and PPV was $100 \%$.

Similarly Karjalainen et al., [12] found a similar sensitivity for MRI of $94 \%$, for patients referred for MRI by orthopaedic surgeons, which is very similar to their findings. These authors also reported a $19 \%$ false positive rate among asymptomatic tendons, which reinforces the caution needed in interpreting MRI findings.

Ibrahim et al., [7] had found that ultrasound was good in the diagnosis of tendinopathy as well as MRI but with MRI they could differentiate the tendinopathy into peri tendinosis, tendinosis and tenosynovitis, however in advanced cases of tendinosis they found it was difficult to differentiate it from partial thickness tear as we depend on the presence of heterogeneous signal intensity of the tendon substance, also five cases of small partial thickness tear could only be diagnosed by MRI but not by ultrasound. In full thickness tear the ultrasound was as accurate as MRI.

\section{Conclusion}

Ultrasound is an important complementary diagnostic tool in the diagnosis of Achilles tendon injuries, it is as good as MRI in the diagnosis of tendinopathy and full thickness tear, however MRI is more superior in the diagnosis of partial thickness tear, and in the differentiation of the different types of tendon injuries.

\section{References}

[1]Z. S. Rosenberg, J. Beltran, and J. T. Bencardino, "MR imaging of the ankle and foot," Radiographics, Vol. 20, PP. S153S179, 2000.

[2] D. N. Garras, S. M. Raikin, S. B. Bhat, N. Taweel, and H. Karanjia, "MRI is unnecessary for diagnosing acute Achilles tendon ruptures: clinical diagnostic criteria," Clin. Orthop. Relat. Res., Vol. 470, PP. 2268-2273, 2012.

[3] M. E. Schweitzer and D. Karasick, "MR imaging of disorders of the Achilles tendon," Am. J. Roentgenol., Vol. 175, PP. 613-625, 2000.

[4] D. P. Fessell et al., "US of the ankle: technique, anatomy, and diagnosis of 
pathologic conditions.," Radiographics, Vol. 18, PP. 325-340, 1998.

[5] M. R. Carmont, "Achilles tendon rupture: the evaluation and outcome of percutaneous and minimally invasive repair," Br J Sport. Med, Vol. 52, PP. 1281-1282, 2018.

[6] P. C. Noback et al., "Prevalence of asymptomatic achilles tendinosis," Foot ankle Int., Vol. 39, PP. 1205-1209, 2018.

[7] N. M. A. Ibrahim and H. H. Elsaeed, "Lesions of the Achilles tendon: Evaluation with ultrasonography and magnetic resonance imaging," Egypt. J. Radiol. Nucl. Med., Vol. 44, PP. 581-587, 2013.

[8] A. Shalabi, "Magnetic resonance imaging in chronic Achilles tendinopathy," Acta radiol., Vol. 45, no. sup432, PP. 1-45, 2004.

[9] T. Movin, M. Kristoffersen-Wiberg, A.
Shalabi, A. Gad, P. Aspelin, and C. Rolf, "Intratendinous alterations as imaged by ultrasound and contrast medium-enhanced magnetic resonance in chronic achillodynia," Foot ankle Int., Vol. 19, PP. 311-317, 1998.

[10] S. Bianchi and C. Martinoli, Ultrasound of the musculoskeletal system. Springer Science \& Business Media, Vol. 9, PP.45 -57, 2007.

[11] P. Hartgerink, D. P. Fessell, J. A. Jacobson, and M. T. van Holsbeeck, "Fullversus partial-thickness Achilles tendon tears: sonographic accuracy and characterization in 26 cases with surgical correlation," Radiology, Vol. 220, PP. 406-412, 2001.

[12] P. T. Karjalainen ,"MR imaging of overuse injuries of the Achilles tendon," Am. J. Roentgenol., Vol. 175, PP. 251-260, 2000. 